\title{
REFINED WING ASYMPTOTICS FOR THE MERTON AND KOU JUMP DIFFUSION MODELS
}

\author{
STEFAN GERHOLD, JOHANNES F. MORGENBESSER, AND AXEL ZRUNEK
}

\begin{abstract}
Refining previously known estimates, we give large-strike asymptotics for the implied volatility of Merton's and Kou's jump diffusion models. They are deduced from call price approximations by transfer results of Gao and Lee. For the Merton model, we also analyse the density of the underlying and show that it features an interesting "almost power law" tail.
\end{abstract}

\section{INTRODUCTION}

In recent years, the literature on asymptotic approximations of option prices and implied volatilities has grown at a fast pace. Important papers on large-strike asymptotics include [2, 3, 11, 13. Of particular relevance to the present note is the approach of Gao and Lee [8], who translate call price asymptotics to implied volatility asymptotics, robustly w.r.t. choice of model and asymptotic regime. So far, relatively few models have been analyzed in sufficient detail to use the full power of their transfer results. We extend concrete applicability of some results of 8 by presenting refined strike asymptotics for the well-known jump diffusion models of Merton [15] and Kou [12. Potential practical consequences of our expansions concern fast calibration and implied volatility parametrization resp. extrapolation.

As we are in a fixed-maturity regime, we can set the interest rate to zero throughout. Also, initial spot is normalized to $S_{0}=1$. Log-returns are modeled by a Lévy jump diffusion

$$
X_{t}=b t+\sigma W_{t}+\sum_{j=1}^{N_{t}} Y_{j}
$$

with drift $b \in \mathbb{R}$ and diffusion volatility $\sigma>0$. The process $W$ is a standard Brownian motion, $N$ is a Poisson process with intensity $\lambda>0$, and the jumps $Y_{j}$ are i.i.d. real random variables. As for the law of the $Y_{j}$, we focus on the double exponential (Kou) and Gaussian (Merton) cases. The (dimensionless) implied volatility $V(k)$ is the solution of

where

$$
c_{\mathrm{BS}}(k, V(k))=\mathbb{E}\left[\left(S_{T}-e^{k}\right)^{+}\right],
$$

$$
c_{\mathrm{BS}}(k, v)=\Phi\left(d_{1}\right)-e^{k} \Phi\left(d_{2}\right)
$$

is the Black-Scholes call price, with $d_{1,2}=-k / v \pm v / 2$ and $\Phi$ the standard Gaussian cdf. Our interest is in the growth order of $V(k)$ as $k \rightarrow \infty$. While first order asymptotics are known for both models we treat, they suffer from limited practical applicability. Higher order terms typically increase accuracy significantly, even for moderate values of the log-strike $k$.

Date: October 31, 2018.

2010 Mathematics Subject Classification. 91G20, 41A60.

Key words and phrases. Implied volatility, jump diffusion, Kou model, Merton model, saddle point method.

This note is based in part on the thesis 16, where some proofs are discussed in greater detail. S. Gerhold gratefully acknowledges financial support from the Austrian Science Fund (FWF) under grant P 24880-N25. 
We observe that the large-strike behavior of the Kou model is of the same shape (in terms of the asymptotic elements involved) as for the Heston model [7]. Not obvious from the respective model dynamics, this fact is an immediate consequence of the local behavior of the moment generating function (mgf) at the critical moments. This behavior was analyzed in [7] from affine principles, whereas the present analysis for the Kou model profits from its very simple explicit mgf.

For the Merton model, we include an approximation of the density (Theorem 7). The reason is that it implies an interesting "almost power law" tail for the marginals of the underlying, of order $k^{-\sqrt{\log \log k}}$.

Throughout the paper, $F(k) \ll G(k)$ means that the functions $F$ and $G$ satisfy $F(k)=O(G(k))$ for $k \rightarrow \infty$.

\section{KOU JUMP DIFFUSION MODEL}

In the Kou model [12], the $Y_{j}$ are double exponentially distributed, and thus have the common density

$$
f(y)=p \lambda_{+} e^{-\lambda_{+} y} \mathbf{1}_{[0, \infty)}(y)+(1-p) \lambda_{-} e^{\lambda_{-} y} \mathbf{1}_{(-\infty, 0)}(y)
$$

with parameters $\lambda_{+}>1, \lambda_{-}>0$ and $p \in(0,1)$. One of the advantages of this model is the memoryless property of the double exponential distribution, which leads to analytical formulas for several types of options. The moment generating function of the log-price $X_{T}$ is given by

$$
\begin{aligned}
M(s, T) & =\mathbb{E}\left[\exp \left(s X_{T}\right)\right] \\
& =\exp \left(T\left(\frac{\sigma^{2} s^{2}}{2}+b s+\lambda\left(\frac{\lambda_{+} p}{\lambda_{+}-s}+\frac{\lambda_{-}(1-p)}{\lambda_{-}+s}-1\right)\right)\right) .
\end{aligned}
$$

From Benaim and Friz' refinement of Lee's moment formula (Example 5.3 in [2]), it is known that $V$ has the first order asymptotics

$$
\lim _{k \rightarrow \infty} \frac{V(k)}{k^{1 / 2}}=\Psi^{1 / 2}\left(\lambda_{+}-1\right)
$$

where $\Psi(x)$ is defined by

$$
\Psi(x)=2-4\left(\sqrt{x^{2}+x}-x\right) .
$$

To formulate our refined expansion for the Kou call price, define

$$
\begin{aligned}
& \alpha_{1}=\lambda_{+}-1, \quad \alpha_{1 / 2}=-2\left(\lambda \lambda_{+} p T\right)^{1 / 2} \\
& \alpha_{0}=T\left(-\frac{\sigma^{2} \lambda_{+}^{2}}{2}-b \lambda_{+}-\frac{\lambda \lambda_{-}(1-p)}{\lambda_{-}+\lambda_{+}}+\lambda\right)-\log \frac{\left(\lambda \lambda_{+} p T\right)^{1 / 4}}{2 \sqrt{\pi} \lambda_{+}\left(\lambda_{+}-1\right)} .
\end{aligned}
$$

For better readability, the notation here $\left(\alpha_{i}\right.$, and $\beta_{i}$ below) is as in Corollary 7.11 of [8]; coefficient indices mimic the asymptotic terms they belong to.

Theorem 1. The price of a call option in the Kou model satisfies

$$
C(k, T)=\exp \left(-\alpha_{1} k-\alpha_{1 / 2} k^{-1 / 2}-\alpha_{0}\right) k^{-3 / 4}\left(1+O\left(k^{-1 / 4}\right)\right)
$$

as $k \rightarrow \infty$.

The call price expansion (4) is amenable to the general transfer results of Gao and Lee [8]. Indeed, their Corollary 7.11 immediately implies the following implied volatility expansion.

Corollary 2. The implied volatiliy of the Kou model satisfies, as $k \rightarrow \infty$,

$$
V(k)=\beta_{1 / 2} k^{1 / 2}+\beta_{0}+\beta_{\ell-1 / 2} \frac{\log k}{k^{1 / 2}}+\beta_{-1 / 2} \frac{1}{k^{1 / 2}}+O\left(k^{-3 / 4}\right),
$$




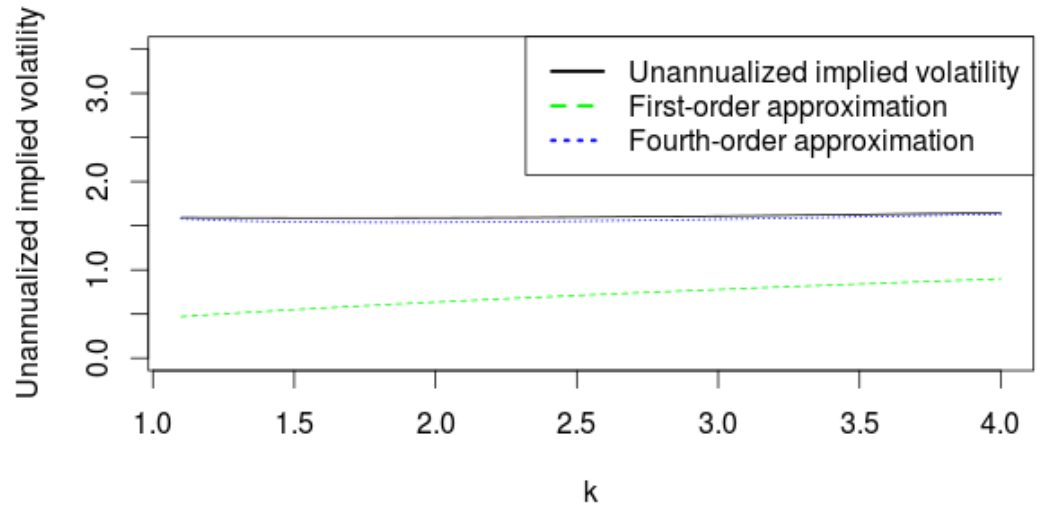

FIGURE 1. Implied volatility of the Kou model (solid) compared with the fourth-order expansion (5) (dotted) and Lee's formula (2) (dashed). The parameters are $T=6, \sigma=0.4, \lambda=1, p=0.2$, $\lambda_{-}=2$, and $\lambda_{+}=3$.

where

$$
\begin{aligned}
\beta_{1 / 2} & =-2 \gamma \sqrt{\alpha_{1}^{2}+\alpha_{1}}=\Psi^{1 / 2}\left(\lambda_{+}-1\right), \\
\beta_{0} & =\gamma \alpha_{1 / 2}, \quad \beta_{\ell-1 / 2}=\frac{\gamma}{4}, \\
\beta_{-1 / 2} & =\left(\alpha_{0}+\log \frac{1-\left(1+\frac{1}{\alpha_{1}}\right)^{-1 / 2}}{\sqrt{4 \pi \alpha_{1}}}\right) \gamma+\left(\frac{1}{2\left(2 \alpha_{1}\right)^{3 / 2}}-\frac{1}{2\left(2 \alpha_{1}+2\right)^{3 / 2}}\right) \alpha_{1 / 2}^{2},
\end{aligned}
$$

and

$$
\gamma=\left(\frac{1}{\sqrt{2 \alpha_{1}+2}}-\frac{1}{\sqrt{2 \alpha_{1}}}\right) .
$$

The gain in numerical precision over the first order approximation (2) depends on the model parameters. See Figures 1 and 2 for examples. It is an interesting fact that the expansion (5) has the same shape as the implied vol expansion of the Heston model (see Theorem 1.3 in [7]). While this is not obvious from the model specifications, it is clear from an asymptotic analysis of the respective mgfs: Call price and density asymptotics are governed by the type of singularity found at the critical moment, and this is "exponential of a first order pole" in both cases. (For other papers analyzing functions with such behavior, see, e.g., 6, 9].)

We briefly comment on the qualitative implications of Corollary 2, The dominating term depends only on $\lambda_{+}$, i.e., the parameter that governs the propensity to jump in the in-the-money direction. If $\lambda_{+}$increases, upward jumps become smaller, and the call clearly becomes cheaper, thus lowering implied volatility. (Note that the function $\Psi$ is decreasing.) Second order asymptotics of implied vol, i.e., $\beta_{0}$ in (5), depend additionally on $\lambda, p$, and $T$. It is remarkable that the smile wings are very insensitive to the diffusion volatility $\sigma$ and the downwards-jump parameter $\lambda_{-}$, which appear only in the $k^{-1 / 2}$-term in (5).

As the transfer from call price to implied vol asymptotics is handled by $[8$ in a mechanical way, the rest of this section is devoted to the proof of Theorem 1. The mgf (1) is analytic in the strip $\Re s \in\left(\lambda_{-}, \lambda_{+}\right)$. By the exponential decay of $M(s, T)$ 


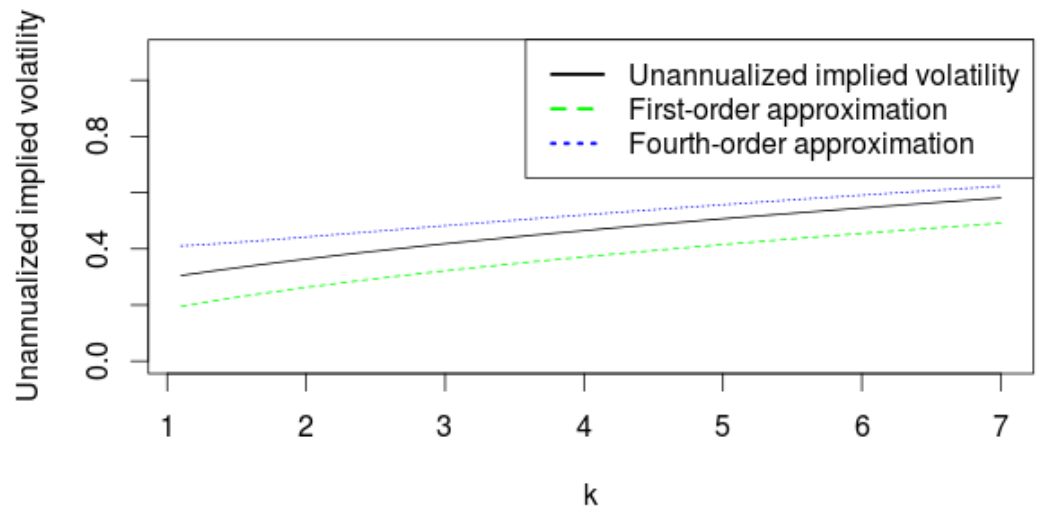

FiguRE 2. Implied volatility of the Kou model (solid) compared with the fourth-order expansion (5) (dotted) and Lee's formula (2) (dashed). The parameters are $T=1, \sigma=0.1, \lambda=5, \lambda_{+}=15$, $\lambda_{-}=15, p=0.5$.

for $|\Im s| \rightarrow \infty$, the Fourier representation

$$
C(k, T)=\frac{e^{k}}{2 \pi i} \int_{\eta-i \infty}^{\eta+i \infty} e^{-k s} \frac{M(s, T)}{s(s-1)} d s
$$

of the call price is valid; see Lee [14]. The real part of the integration contour satisfies $1<\eta<\lambda_{+}$. We prove Theorem 1 by a saddle point analysis of 6 ). (The series representation of the call price from Kou's paper 12 seems less amenable to asymptotic analysis.) The integrand blows up as $s \rightarrow \lambda_{+}$. Identifying the dominating term $1 /\left(\lambda_{+}-s\right)$ in $(1)$, we define the (approximate) saddle point $\hat{s}=$ $\hat{s}(k)$ as the solution of

which is given by

$$
\frac{\partial}{\partial s} e^{-k s} \exp \left(T \lambda \frac{\lambda_{+} p}{\lambda_{+}-s}\right)=0,
$$

where $\xi=\lambda \lambda_{+} p T$.

Lemma 3. The cumulant generating function $m(s, T)=\log M(s, T)$ of $X_{T}$ satisfies

$$
\begin{aligned}
m(\hat{s}, T) & =\frac{T \sigma^{2} \lambda_{+}^{2}}{2}+b \lambda_{+} T+\xi^{1 / 2} k^{1 / 2}+\frac{T \lambda \lambda_{-}(1-p)}{\lambda_{-}+\lambda_{+}}-\lambda T+O\left(k^{-1 / 2}\right), \\
m^{\prime}(\hat{s}, T) & =k+O(1), \\
m^{\prime \prime}(\hat{s}, T) & =2 \xi^{-1 / 2} k^{3 / 2}+O(1), \\
m^{\prime \prime \prime}(\hat{s}+i t, T) & =O\left(k^{2}\right) \quad \text { for }|t|<k^{-\alpha}, \alpha>0,
\end{aligned}
$$

where all derivatives are with respect to $s$.

Proof. The estimates follow by straightforward computations from (1).

We now move the integration contour so that it passes through the saddle point $\hat{s}$. A small part of the contour, within distance $O\left(k^{-\alpha}\right)$ of the saddle point, captures 
the asymptotics of the full integral. Any exponent $\alpha \in\left(\frac{2}{3}, \frac{3}{4}\right)$ is suitable. The integration variable thus becomes $s=\hat{s}+i t,|t|<k^{-\alpha}$. From the estimates for $m^{\prime}$ and $m^{\prime \prime \prime}$ in Lemma 3 , we have the local expansion

$$
M(\hat{s}+i t, T)=\exp \left(m(\hat{s}, T)+i t k-\frac{m^{\prime \prime}(\hat{s}, T)}{2} t^{2}\right)\left(1+O\left(t+t^{3} k^{2}\right)\right) .
$$

The rational function $1 /(s(s-1))$ is locally constant, to first order:

$$
\frac{1}{(\hat{s}+i t)(\hat{s}+i t-1)}=\frac{1}{\lambda_{+}\left(\lambda_{+}-1\right)}\left(1+O\left(k^{-1 / 2}\right)\right) .
$$

The integral close to the saddle point thus becomes

$$
\begin{aligned}
\frac{e^{k}}{2 \pi i} \int_{\hat{s}-i k^{-\alpha}}^{\hat{s}+i k^{-\alpha}} & e^{-k s} \frac{M(s, T)}{s(s-1)} d s \\
& =\frac{e^{k(1-\hat{s})} M(\hat{s}, T)}{2 \pi \lambda_{+}\left(\lambda_{+}-1\right)} \int_{-k^{-\alpha}}^{k^{-\alpha}} \exp \left(-\frac{m^{\prime \prime}(\hat{s}, T)}{2} t^{2}\right)\left(1+O\left(k^{-3 \alpha+2}\right)\right) d t .
\end{aligned}
$$

Setting $u:=m^{\prime \prime}(\hat{s}, T)^{1 / 2}$, we get from Lemma 3

$$
u=\frac{\sqrt{2} k^{3 / 4}}{\xi^{1 / 4}}\left(1+O\left(k^{-3 / 2}\right)\right) \text { and } \quad \frac{1}{u}=\frac{\xi^{1 / 4}}{\sqrt{2} k^{3 / 4}}\left(1+O\left(k^{-3 / 2}\right)\right) .
$$

By substituting $\omega=u t$ and using the fact that Gaussian integrals have exponentially decaying tails, we find

$$
\begin{aligned}
\int_{-k^{-\alpha}}^{k^{-\alpha}} \exp \left(-\frac{m^{\prime \prime}(\hat{s}, T)}{2} t^{2}\right) d t & =\frac{1}{u} \int_{-u k^{-\alpha}}^{u k^{-\alpha}} \exp \left(-\frac{\omega^{2}}{2}\right) d \omega \\
& =\sqrt{\pi} k^{-3 / 4} \xi^{1 / 4}\left(1+O\left(k^{-3 / 2}\right)\right) .
\end{aligned}
$$

Use this in (7), and replace $M(\hat{s}, T)$ by the estimates in Lemma 3 , to get the right hand side of (4). Note that to obtain a relative error $k^{-1 / 4}$, and not just $k^{-1 / 4+\varepsilon}$, it suffices to take one further term in the local expansion, keeping in mind the wellknown fact that the saddle point method usually yields a full asymptotic expansion. See 7 for a detailed discussion of this issue in a similar analysis. (The same remark applies in case of the Merton model below.) To prove Theorem 1, it thus remains to show that the integral over $|t|>k^{-\alpha}$ can be dropped, so that (7) asymptotically equals (6). This tail estimate is done in the following lemma. By symmetry, it suffices to treat $t>k^{-\alpha}$.

Lemma 4. Let $\frac{2}{3}<\alpha<\frac{3}{4}$. Then we have

$$
\frac{e^{k}}{2 \pi i} \int_{\hat{s}+i k^{\alpha}}^{\hat{s}+i \infty} e^{-k s} \frac{M(s, T)}{s(s-1)} d s \ll e^{k\left(1-\lambda_{+}\right)+2 \xi^{1 / 2} k^{1 / 2}-\xi^{-1 / 2} k^{3 / 2-2 \alpha} / 2} .
$$

Proof. Let $s=\hat{s}+i t=\lambda_{+}-\xi^{1 / 2} k^{-1 / 2}+i t$, where $t \geq k^{-\alpha}$. Then

$$
\begin{aligned}
|M(s, T)| & \ll \exp \left(\Re\left(\frac{\xi}{\xi^{1 / 2} k^{-1 / 2}-i t}\right)\right) \\
& =\exp \left(\frac{\xi^{1 / 2} k^{1 / 2}}{1+t^{2} \xi^{-1} k}\right) \ll \exp \left(\frac{\xi^{1 / 2} k^{1 / 2}}{1+k^{1-2 \alpha} \xi^{-1}}\right) .
\end{aligned}
$$

Using the fact $1 /(1+x) \leq 1-x / 2$ for $x \leq 1$ and that $k^{1-2 \alpha}$ is smaller than 1 for sufficiently large $k$, we get

$$
|M(s, T)| \ll \exp \left(\xi^{1 / 2} k^{1 / 2}-\xi^{-1 / 2} k^{3 / 2-2 \alpha} / 2\right) .
$$


From $|s(s-1)| \gg 1+t^{2}$ and $(9)$, we obtain

$$
\frac{e^{k}}{2 \pi}\left|e^{-k s} \frac{M(s, T)}{s(s-1)}\right| \ll \frac{e^{k\left(1-\lambda_{+}\right)+2 \xi^{1 / 2} k^{1 / 2}-\xi^{-1 / 2} k^{3 / 2-2 \alpha} / 2}}{1+t^{2}}
$$

and thus

$$
\begin{aligned}
\frac{e^{k}}{2 \pi i} \int_{\hat{s}+i k^{-\alpha}}^{\hat{s}+i \infty} e^{-k s} \frac{M(s, T)}{t(s-1)} d s & \ll e^{k\left(1-\lambda_{+}\right)+2 \xi^{1 / 2} k^{1 / 2}-\xi^{-1 / 2} k^{3 / 2-2 \alpha} / 2} \int_{k^{-\alpha}}^{\infty} \frac{d t}{1+t^{2}} \\
& \ll e^{k\left(1-\lambda_{+}\right)+2 \xi^{1 / 2} k^{1 / 2}-\xi^{-1 / 2} k^{3 / 2-2 \alpha} / 2} .
\end{aligned}
$$

The proof of Theorem 1 is complete. Finally, we mention that tail asymptotics for the distribution of $X_{T}$ (for $\sigma^{2}=0$ ) can be found in [1], Example 7.5. They can also be deduced from earlier work of Embrechts et al. [5].

\section{MERTON JUMP DIFFUSION MODEL}

In one of his classical papers, Merton [15] proposed a Lévy jump diffusion with Gaussian jumps as a model for log-returns. If mean and variance of the jump size distribution are $\mu$ resp. $\delta^{2}$, then the mgf is the entire function

$$
M(s, T)=\exp \left(T\left(\frac{1}{2} \sigma^{2} s^{2}+b s+\lambda\left(e^{\delta^{2} s^{2} / 2+\mu s}-1\right)\right)\right) .
$$

Benaim and Friz [3] gave first order logarithmic asymptotics for the call price,

$$
L:=-\log C(k, T) \sim \frac{\sqrt{2}}{\delta} k \sqrt{\log k}, \quad k \rightarrow \infty,
$$

and first order asymptotics for implied volatility:

$$
V(k) \sim 2^{-3 / 4} \sqrt{\delta k} /(\log k)^{1 / 4} .
$$

Our refined results are best formulated using the implicitly defined saddle point $\hat{s}=$ $\hat{s}(k)$, satisfying $m^{\prime}(\hat{s}, T)=k$. (As in the Kou model, we write $m=\log M$ for the cumulant generating function.)

Theorem 5. For $k \rightarrow \infty$, the call price in the Merton model satisfies

$$
\begin{aligned}
C(k, T)= & \frac{\delta^{2} e^{k(1-\hat{s})} M(\hat{s}, T)}{2 \log k \sqrt{2 \pi m^{\prime \prime}(\hat{s}, T)}}\left(1+O\left(\frac{1}{\sqrt{\log k}}\right)\right) \\
= & \frac{\delta^{2} e^{k(1-\hat{s})+T}\left(\sigma^{2} \hat{s}^{2} / 2+b \hat{s}+\lambda\left(e^{\delta^{2} \hat{s}^{2} / 2+\mu \hat{s}}-1\right)\right)}{2 \log k \sqrt{2 \pi T\left(\sigma^{2}+\lambda\left(\delta^{2} \hat{s}+\mu\right)\left(\left(\delta^{2} \hat{s}+\mu\right)+\delta^{2}\right) e^{\delta^{2} \hat{s}^{2} / 2+\mu \hat{s}}\right)}} \\
& \cdot\left(1+O\left(\frac{1}{\sqrt{\log k}}\right)\right) .
\end{aligned}
$$

Corollary 6. The implied volatility of the Merton model satisfies, for $k \rightarrow \infty$,

$$
\begin{aligned}
& V(k)=G_{-}\left(k, L-\frac{3}{2} \log L+\log \frac{k}{4 \sqrt{\pi}}\right)+O\left(k^{-1 / 2}(\log k)^{-5 / 4}\right) \\
& =2^{-3 / 4} \sqrt{\delta k}(\log k)^{-1 / 4}+c \sqrt{k}(\log k)^{-3 / 4}+O\left(\frac{\sqrt{k} \log \log k}{(\log k)^{5 / 4}}\right),
\end{aligned}
$$

where $L=-\log C(k, T)$ is the absolute log of the call price, $G_{-}(k, u)=\sqrt{2}(\sqrt{u+k}-$ $\sqrt{u})$, and $c=2^{-9 / 4} \delta^{-1 / 2}\left(\mu+\delta^{2}\right)-2^{-13 / 4} \delta^{3 / 2}$. 


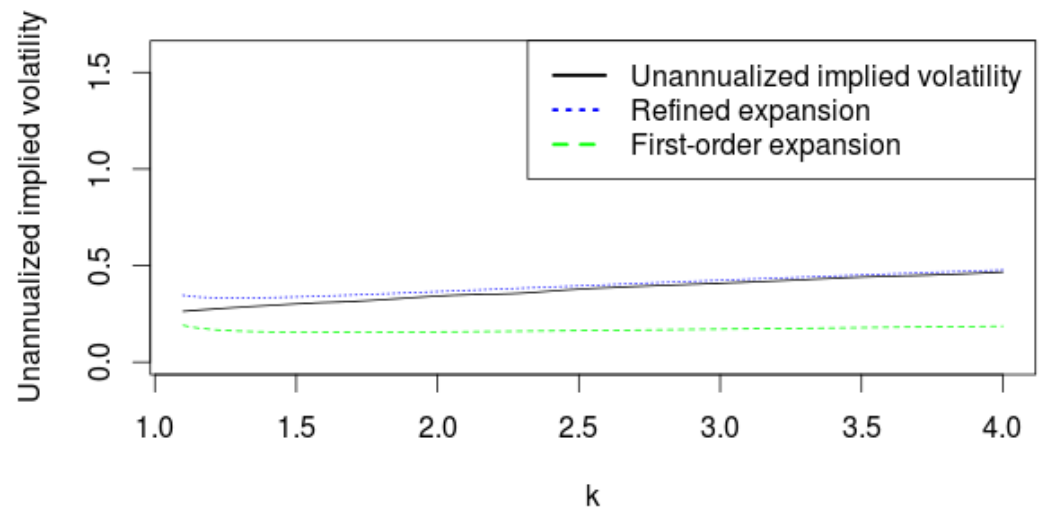

Figure 3. The solid curve is the implied vol of the Merton model, with parameters $T=0.1, \sigma=0.4, \lambda=0.1, \mu=0.3$, and $\delta=0.4$. The dashed line is the first order approximation $(11)$, whereas the dotted line is our refined approximation (14).

Theorem 7. The density of the Merton log-return $X_{T}$ satisfies, for $k \rightarrow \infty$,

$$
\begin{aligned}
f_{X_{T}}(k) & =\frac{e^{-k \hat{s}} M(\hat{s}, T)}{\sqrt{2 \pi m^{\prime \prime}(\hat{s}, T)}}\left(1+O\left(k^{-1 / 2}\right)\right) \\
& =\frac{e^{-k \hat{s}+T\left(\sigma^{2} / 2 \hat{s}^{2}+b \hat{s}+\lambda\left(e^{\delta^{2} / 2 \hat{s}^{2}+\mu \hat{s}}-1\right)\right)}}{\sqrt{2 \pi T\left(\sigma^{2}+\lambda\left(\delta^{2} \hat{s}+\mu\right)\left(\left(\delta^{2} \hat{s}+\mu\right)+\delta^{2}\right) e^{\delta^{2} \hat{s}^{2} / 2+\mu \hat{s}}\right)}}\left(1+O\left(k^{-1 / 2}\right)\right) \\
& =\exp \left(-\frac{\sqrt{2}}{\delta} k \sqrt{\log k}+O(k)\right)
\end{aligned}
$$

Formula (13) is just (12) with $M$ replaced by its explicit form, and the same holds for (17) and (16). For numerical accuracy, it is preferable to use (14) and (17), and not the more explicit variants $(15)$ and 18 .

We begin the proofs by deducing 15$)$ and $(18)$ from the semi-explicit formulas (14) and (17), using asymptotic approximations of $\hat{s}$. The saddle point satisfies

$$
\hat{s}=\frac{\sqrt{2 \log k}}{\delta}-\frac{\mu}{\delta^{2}}+O\left(\frac{\log \log k}{\sqrt{\log k}}\right) .
$$

This estimate follows from the saddle point equation $m^{\prime}(\hat{s}, T)=k$ by a tedious, but straightforward application of the classical "bootstrapping" technique (see, e.g., chapter 22 of [10]). From the saddle point equation, we know that

$$
e^{\delta^{2} \hat{s}^{2} / 2+\mu \hat{s}}=\frac{k / T-\sigma^{2} \hat{s}-b}{\lambda\left(\delta^{2} \hat{s}+\mu\right)} \sim \frac{1}{\lambda \delta T \sqrt{2}} \frac{k}{\sqrt{\log k}} .
$$

Using these properties in (17) yields (18). For the density of the underlying itself we thus obtain

$$
f_{S_{T}}(k)=f_{X_{T}}(\log k) / k=k^{-(\sqrt{2} / \delta) \sqrt{\log \log k}} \cdot e^{O(\log k)} .
$$


The marginal law of the underlying has "almost" a power law tail, due to the very slow increase of $\sqrt{\log \log k}$, but it is still asymptotically lighter than that of any power law. The influence of the model parameters seems a bit surprising here: Neither the jump size nor the Poisson intensity appear in the main factor in (21), but only the jump size variance.

To obtain the explicit refined volatility expansion $(15)$, note that, by $(13),(19)$, and $(20)$, we can refine 10 to

$$
L=\frac{\sqrt{2}}{\delta} k \sqrt{\log k}-\frac{\mu+\delta^{2}}{\delta^{2}} k+O\left(\frac{k \log \log k}{\sqrt{\log k}}\right) .
$$

Since

$$
G_{-}(k, u)=\frac{\sqrt{2}}{2} k u^{-1 / 2}-\frac{\sqrt{2}}{8} k^{2} u^{-3 / 2}+O\left(k^{3} u^{-5 / 2}\right),
$$

using 22 in 14 yields 15 .

We proceed to the proofs of the first equalities in Theorem 5 and Corollary 6 . The proof of Theorem 7 is very similar to that of Theorem 5 , using the Fourier representation of the density, and is omitted (see [16]). (Alternatively, the density asymptotics could be deduced from its series representation, (4.12) in [4, by the Laplace method.) Formula (14) in Corollary 6 is a special case of Corollary 7.1 in 8, the error term follows from (10). Our Theorem 5 is then useful for approximating $L$ in (14) numerically, or symbolically to obtain the explicit expansion (15); see above. Note that our refined call approximation yields the second order term (and higher order terms as well) in (15), which cannot be be deduced from $(10)$.

It remains to prove (12). Again, we appeal to the representation (6), where $\eta>1$, and shift the integration contour to the saddle point $\hat{s}$. For the central range, we let $s=\hat{s}+i t$, where $|t|<k^{-\alpha}$ with $\alpha \in\left(\frac{1}{3}, \frac{1}{2}\right)$.

Lemma 8. The cumulant generating function $m(s, T)=\log M(s, T)$ satisfies for, $k \rightarrow \infty$,

$$
\begin{aligned}
m(\hat{s}, T) & =\frac{k}{\delta \sqrt{2 \log k}}\left(1+O\left(\frac{\log \log k}{\log k}\right)\right), \\
m^{\prime}(\hat{s}, T) & =k, \\
m^{\prime \prime}(\hat{s}, T) & =\delta k \sqrt{2 \log k}\left(1+O\left(\frac{1}{\sqrt{\log k}}\right)\right), \\
m^{\prime \prime \prime}(\hat{s}+i t, T) & =2 k \log k\left(1+O\left(\frac{1}{\log k}\right)\right),
\end{aligned}
$$

where $|t|<k^{-\alpha}$.

Proof. As for the Kou model, these expansions follow by a straightforward computation from the explicit mgf. For the second equation, note that we are using the exact saddle point, and not an approximation as we did in the Kou model.

Since the saddle point tends to infinity, the rational function $1 /(s(s-1))$ locally tends to zero:

$$
\frac{1}{(\hat{s}+i t)(\hat{s}-1+i t)}=\frac{\delta^{2}}{2 \log k}\left(1+O\left(\frac{1}{\sqrt{\log k}}\right)\right) .
$$

(We have used (19) here.) Using this and Lemma 8, we see that the integral over the central range has the asymptotics $\sqrt{12}$, , after handling the Gaussian integral as in (8). To complete the proof, we need to provide a tail estimate, to the effect that the integral outside of $s=\hat{s}+i t$ with $|t|<k^{-\alpha}$ is negligible. Again, it suffices to treat the upper portion, by symmetry. 
Lemma 9. Let $\frac{1}{3}<\alpha<\frac{1}{2}$. Then we have

$$
\frac{e^{k}}{2 \pi i} \int_{\hat{s}+i k^{-\alpha}}^{\hat{s}+i \infty} \frac{e^{-k s} M(s, T)}{s(s-1)} d s \ll \frac{e^{k(1-\hat{s})} M(\hat{s}, T) e^{-\delta k^{1-2 \alpha} /(2 \sqrt{2 \log k})}}{\log k} .
$$

Proof. We first bound $M(s, T)$ :

$$
\begin{aligned}
|M(s, T)| & =\exp \left(\Re\left(T\left(\frac{\sigma^{2}}{2} s^{2}+b s+\lambda\left(e^{\delta^{2} s^{2} / 2+\mu s}-1\right)\right)\right)\right) \\
& =\exp \left(T\left(\frac{\sigma^{2}}{2}\left(\hat{s}^{2}-t^{2}\right)+b \hat{s}+\lambda\left(\cos \left(\delta^{2} t \hat{s}+\mu t\right) e^{\delta^{2}\left(\hat{s}^{2}-t^{2}\right) / 2+\mu \hat{s}}-1\right)\right)\right) \\
& \leq \exp \left(T\left(\frac{\sigma^{2}}{2}\left(\hat{s}^{2}-t^{2}\right)+b \hat{s}+\lambda\left(e^{\delta^{2} \hat{s}^{2} / 2+\mu \hat{s}} e^{-\delta^{2} k^{-2 \alpha} / 2}-1\right)\right)\right) .
\end{aligned}
$$

Using

$$
e^{-\delta^{2} k^{-2 \alpha} / 2}=1-\delta^{2} k^{-2 \alpha} / 2+O\left(k^{-4 \alpha}\right)
$$

and 20, we get

$$
M(s, T) \ll e^{-T \sigma^{2} t^{2} / 2} M(\hat{s}, T) e^{-\delta k^{1-2 \alpha} /(2 \sqrt{2 \log k})} .
$$

Since $|s(s-1)| \gg \log k$, this estimate implies

$$
\begin{aligned}
\frac{e^{k}}{2 \pi i} \int_{\hat{s}+i k^{-\alpha}}^{\hat{s}+i \infty} \frac{e^{-k s} M(s, T)}{s(s-1)} d s & \ll \frac{e^{k(1-\hat{s})} M(\hat{s}, T) e^{-\delta k^{1-2 \alpha} /(2 \sqrt{2 \log k})}}{\log k} \int_{k^{-\alpha}}^{\infty} e^{-\sigma^{2} t^{2} T / 2} d t \\
& \ll \frac{e^{k(1-\hat{s})} M(\hat{s}, T) e^{-\delta k^{1-2 \alpha} /(2 \sqrt{2 \log k})}}{\log k} .
\end{aligned}
$$

\section{REFERENCES}

[1] J. M. P. Albin And M. Sundén, On the asymptotic behaviour of Lévy processes. I. Subexponential and exponential processes, Stochastic Process. Appl., 119 (2009), pp. 281-304.

[2] S. Benaim And P. Friz, Smile asymptotics II: Models with known moment generating functions, J. Appl. Probab., 45 (2008), pp. 16-32.

[3] S. Benaim And P. Friz, Regular variation and smile asymptotics, Math. Finance, 19 (2009), pp. $1-12$.

[4] R. Cont And P. TAnkov, Financial modelling with jump processes, Chapman \& Hall/CRC Financial Mathematics Series, Chapman \& Hall/CRC, Boca Raton, FL, 2004.

[5] P. Embrechts, J. L. Jensen, M. Maejima, and J. L. Teugels, Approximations for compound Poisson and Pólya processes, Adv. in Appl. Probab., 17 (1985), pp. 623-637.

[6] P. Flajolet, S. Gerhold, And B. Salvy, Lindelöf representations and (non-)holonomic sequences, Electronic Journal of Combinatorics, 17 (2010).

[7] P. Friz, S. Gerhold, A. Gulisashvili, and S. Sturm, On refined volatility smile expansion in the Heston model, Quantitative Finance, 11 (2011), pp. 1151-1164.

[8] K. Gao And R. LeE, Asymptotics of implied volatility to arbitrary order. Preprint, available at http://ssrn.com/abstract=1768383, 2011.

[9] S. Gerhold, Counting finite languages by total word length, INTEGERS, 11 (2011).

[10] R. L. Graham, M. Grötschel, and L. Lovász, eds., Handbook of combinatorics. Vol. 1, 2, Elsevier Science B.V., Amsterdam, 1995.

[11] A. Gulisashvili, Asymptotic formulas with error estimates for call pricing functions and the implied volatility at extreme strikes, SIAM J. Financial Math., 1 (2010), pp. 609-641.

[12] S. Kou, A jump-diffusion model for option pricing, Management Science, 48 (2002), pp. 10861101.

[13] R. W. LEE, The moment formula for implied volatility at extreme strikes, Math. Finance, 14 (2004), pp. 469-480.

[14] - Option pricing by transform methods: Extensions, unification, and error control, Journal of Computational Finance, 7 (2004), pp. 51-86.

[15] R. C. Merton, Option pricing when underlying stock returns are discontinuous, J. Financial Economics, 3 (1976), pp. 125-144. 
[16] A. ZRUneK, Volatility smile expansions in Lévy models, master's thesis, Vienna University of Technology, December 2013.

Vienna University of Technology, Wiedner Hauptstrasse 8-10/105-1, A-1040 Vienna, Austria

E-mail address: sgerhold@fam.tuwien.ac.at

University of Vienna, Oskar-Morgenstern-Platz 1, A-1090 Vienna, Austria

E-mail address: johannes.morgenbesser@univie.ac.at

E-mail address: axel.zrunek@aon.at 